\title{
Planning and task management in Parkinson's disease: Differential emphasis in dual-task performance
}

\author{
ELLEN BIALYSTOK, ${ }^{1,2}$ FERGUS I.M. CRAIK, ${ }^{2,3}$ AND TARESA STEFURAK ${ }^{3,4}$ \\ ${ }^{1}$ Department of Psychology, York University, Toronto, Ontario, Canada \\ ${ }^{2}$ Rotman Research Institute, Baycrest, Toronto, Ontario, Canada \\ ${ }^{3}$ University of Toronto, Toronto, Ontario, Canada \\ ${ }^{4}$ Toronto East General Hospital, Toronto, Ontario, Canada
}

(Received July 19, 2007; Final Revision October 22, 2007; Accepted October 25, 2007)

\begin{abstract}
Seventeen patients diagnosed with Parkinson's disease completed a complex computer-based task that involved planning and management while also performing an attention-demanding secondary task. The tasks were performed concurrently, but it was necessary to switch from one to the other. Performance was compared to a group of healthy age-matched control participants and a group of young participants. Parkinson's patients performed better than the age-matched controls on almost all measures and as well as the young controls in many cases. However, the Parkinson's patients achieved this by paying relatively less attention to the secondary task and focusing attention more on the primary task. Thus, Parkinson's patients can apparently improve their performance on some aspects of a multidimensional task by simplifying task demands. This benefit may occur as a consequence of their inflexible exaggerated attention to some aspects of a complex task to the relative neglect of other aspects.

(JINS, 2008, 14, 257-265.)
\end{abstract}

Keywords: Parkinsonian disorders, Aging, Task performance, Attention, Set (psychology), Short-term memory

\section{INTRODUCTION}

It is generally agreed that patients with Parkinson's disease (PD) exhibit cognitive deficits on tasks that measure executive control functions (Dirnberger et al., 2005; Dubois \& Pillon, 1997; McNamara et al., 2003; Muslimovic et al., 2005). In particular, PD patients have problems with planning (Johnson et al., 2005; Lewis et al., 2003; Owen et al., 1995), prospective memory (Kliegel et al., 2005), working memory (Dagher et al., 2001; Lewis et al., 2005) and setshifting (Cools et al., 2001; Gauntlett-Gilbert et al., 1999; Woodward et al., 2002). These deficits have been demonstrated using a wide variety of tasks, a common one being the Tower of London along with adaptations and expansions of it that included novel conditions (Culbertson et al., 2004; Dagher et al., 2001; Owen et al., 1995). However, studies typically report performance on a single task or a battery of individual tasks, each of which is designed to isolate only one of these components. This, of course, is the

Correspondence and reprint requests to: Professor Ellen Bialystok, Department of Psychology, York University, 4700 Keele Street, Toronto, Ontario, Canada, M3J 1P3. E-mail: ellenb@yorku.ca only approach by which reliable estimates of the potential impairment in specific processes can be made. The consequence, however, is that there is a dearth of data indicating how PD patients perform in more integrated situations that combine these component processes in a more ecologically valid context.

In an interesting parallel, all the cognitive inefficiencies described earlier have also been found in older adults during normal aging (Braver \& Barch, 2002; Daniels et al., 2006; Robbins et al., 1998), and several researchers have explicitly suggested that age-related dopamine depletion is one main contributor to cognitive dysfunction in the elderly (Braver \& Barch, 2002; Li et al., 2000; Rubin, 1999). In an earlier study, we assessed the abilities of older and younger adults to perform a complex task involving planning, working memory, and executive control; the task simulated cooking breakfast and was ecologically valid and interesting to carry out (Craik \& Bialystok, 2006). This computer-based task required participants to "cook" a breakfast consisting of five foods and concurrently set as many table places as possible without interfering with the accuracy of the cooking demands. The study included three conditions that var- 
ied in difficulty as the information for the cooking activity and the table setting appeared either on a single screen or on different screens that required switching back and forth. All of the actions were carried out by touching images on a touch-screen monitor. The data measured various aspects of executive functioning, including prospective memory, through remembering to start and stop foods at appropriate times; working memory, through holding a general plan in mind; planning, through formulating an effective strategy; co-ordination, through putting the plan into action; and perseveration, through continuing to spend time performing inappropriate activities. The participants were younger $(20$ years) and older (70 years) adults who were monolingual or bilingual. The general finding was that older participants performed more poorly than younger ones on all measures, especially in terms of the planning functions and accuracy of the food cooking. The only difference between the language groups was that the bilinguals were better able to switch between the two tasks and therefore spent less inappropriate time at table setting than the monolinguals when one of the foods required attention.

Some evidence for the validity of the breakfast task comes from a study of older adults reported by Edwards and Ryan (2004). Participants prepared a simple meal in their own home while setting two places at the table by following a set of directions. The activity took place while a talk radio station was playing and a telephone call was received to provide interference. One measure was the number of strategies used to complete the task components and switch attention from one step to the next. In a second session, the participants performed the computerized task used in the present study. The results showed that the number of strategies in the real-life task correlated with several of the measures in the computerized task, including deviation from ideal start times, range of stop times, number of food checks, and number of place settings, all $r \mathrm{~s}>.50$.

Given the proposed similarities between mild PD and normal aging, we studied a group of PD patients performing the breakfast task. Our expectation was that the patients' performance would resemble that of older adults, and that both groups would perform less well than young adults. Since the patients in our study were aged between 60 and 85 , our prediction was that the PD group would be more impaired than age-matched controls on aspects of the task requiring integration of information from several sources and also switching between two concurrent subtasks. In our view, the breakfast task offers a means of establishing a more complete description of the impairments in planning and executive control that are observed in patients with Parkinson's disease.

\section{METHOD}

\section{Participants}

The study included 17 idiopathic Parkinson's patients without dementia ( 9 men, 8 women) with a mean age of 67.5
(6.0) years, 15 age-matched controls ( 5 men, 10 women) with a mean age of 70.3 (4.4) years, and 15 younger controls (5 men, 10 women) with a mean age of 20.5 (2.7) years. The PD patients were recruited from a group attending an out-patient physiotherapy clinic at Baycrest Hospital in Toronto. The two control groups were the monolingual participants reported in the previous study (Craik \& Bialystok, 2006); the younger adults were university students, and the older adults were community-living volunteers. All participants were reimbursed for travel expenses and given a small honorarium for participating.

All of the PD patients were receiving medication for their condition and were medicated at the time of testing. Medications included levodopin, amantadine, sinemet, carbidopa, requip and mirapex, as well as combinations of these for some patients. All patients were informally rated as mentally competent by the RA running the study, and all participants understood the complex task perfectly well. HoehnYahr scores were available for 12 of the PD patients; they varied between stage $1(n=3)$, stage $2(n=3)$, and stage 3 $(n=6)$. MMSE scores were available for 13 of the 17 PD patients; scores ranged from 26 to 30 with a mean of 28.8 . Further confirmation of their intellectual competence is provided by scores on the background tasks (see Results). Several patients had a slight tremor, but all were able to perform the breakfast task without difficulty (see Results). The patients had been diagnosed with PD an average of 5.1 (2.6) years earlier, with a range of diagnoses between 2 and 10 years prior to the study.

All data were obtained in compliance with ethical regulations of York University and Rotman Research Institute of Baycrest.

\section{Background Tasks}

Three tasks assessing background skills were administered to all participants. These three tasks, digit span, alpha span, and Mill Hill Vocabulary had been given to the younger and older adults in the previous study. In addition to allowing comparisons in cognitive ability among the groups, they also provided a general indication of mental competence for the PD patients, although Mill Hill Vocabulary scores are more reflective of premorbid than current cognitive status. The first task, digit span, assessed short-term verbal memory. The experimenter read a list of single-digit numbers (for example, 5-4-8), at the rate of one digit per second, and the participant recited back the digits in the same order. The task began with strings of three digits and presented two trials at each digit length until the participant failed to recall both lists of a particular length. The score was the maximum length for which at least one trial was correct.

In the second task, alpha span, the experimenter read a list of words in a fixed random order (for example, "chairzebra-apple") and the participant attempted to recite the list back in alphabetical order ("apple-chair-zebra"). The task was administered and scored in the same way as the digit 
span test. The alpha span task (Craik, 1986) is a measure of working memory because the items must be stored briefly and then re-ordered mentally before responding.

The third test, the Mill Hill Vocabulary Scale (Raven, 1960), is a multiple-choice test of receptive vocabulary. There are 33 test items, each presented with six response choices and participants choose the response that most closely resembles the target word in meaning. One point is awarded for each correct choice, yielding a maximum score of 33 .

\section{The Breakfast Task}

The breakfast task was implemented on a PC computer using a $34 \mathrm{~cm}$ by $27 \mathrm{~cm}$ touch-screen monitor. The task was to prepare a virtual breakfast consisting of five foods and set a table while the foods were cooking. Each food had a different cooking time, ranging from one minute to four and a half minutes. A START and STOP button was located above and below each food icon, and time was displayed on a thermometer indicating the passage of real time (see Fig. 1). Each thermometer was initially set at the cooking time for its specific food (e.g., $2.5 \mathrm{~min}$ for eggs, $4.5 \mathrm{~min}$ for sausages, Fig. 1a), and each thermometer moved down in real time once its start button was pressed. After the thermometer reached zero (the correct cooking time), the clock continued but there was no indication of the amount of overcooking. When the stop button was pressed the program recorded how long the food had been cooked as well as the duration of undercooking (if stopped before zero) or overcooking (if stopped after zero). At the same time, participants completed a table-setting task in which forks, knives, spoons, and plates that were lined up at the bottom of the screen had to be placed in the correct arrangement in four place settings around a square table (Fig. 1). The places were set by touching an item and then touching its destination on the table. When all four place settings were complete, the items returned to the bottom of the screen and participants continued to set four new place settings. Participants were told to set as many places as they could and that part of their success would be determined by the number of places set. However, it was made clear that successful cooking of the meal was the primary task, and that the main criteria for success were to avoid burning the food and to have all the foods ready at the same time.

Complexity was manipulated by varying the number of screens needed to complete both tasks. In the 1-screen condition, all the foods and the table were on the same screen,

(a)

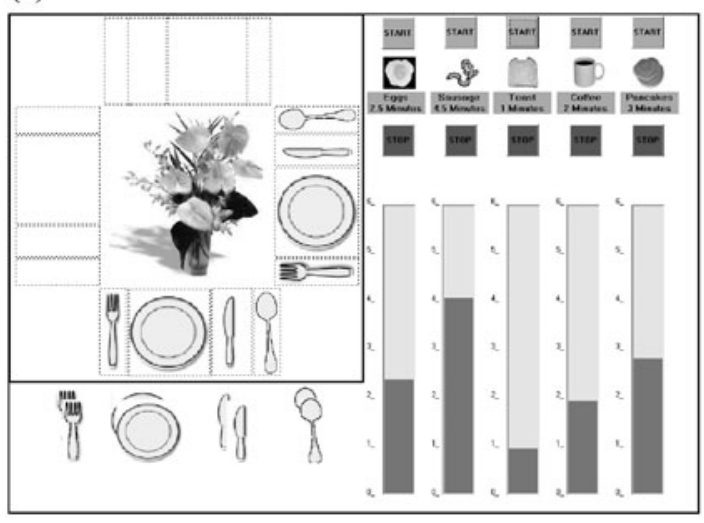

(b)

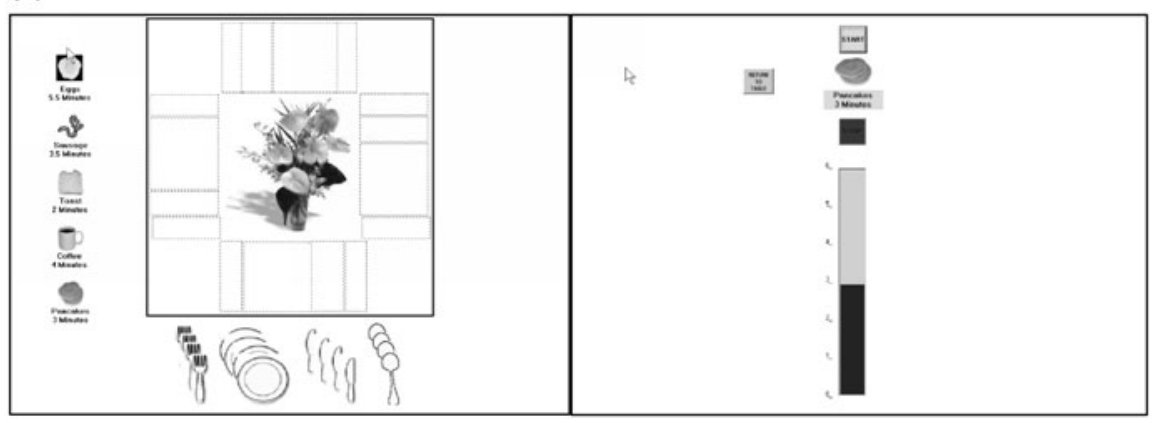

Fig. 1. (a) Sample screen for 1-screen condition of Breakfast Task, showing foods in process of being cooked and table partly set. Neither the toast nor coffee has been started, so their cooking indicators remain at 1 and 2 min respectively. The eggs and pancakes have each been cooking for a few seconds, and the sausages have been cooking for almost 30 s. (b) Sample of two screens for the 6-screen condition showing the main panel with the table on the left and one of the foods and its timer on the right. 
as shown in Figure 1a. In the 2-screen condition, the table appeared on one screen and the five foods and their time bars appeared on a different screen, requiring switching between the two tasks. In the 6-screen condition, the table screen was similar to that in the 2 -screen condition, but each of the foods was on a separate screen (Fig. 1b), requiring switching between the five cooking items as well as between the cooking and table-setting tasks. The participant switched to a cooking screen by pressing one of the food icons to the left of the table, and then back to the table screen by pressing the button RETURN TO TABLE to the left of the food thermometer (Fig. 1b).

The task began with detailed instructions and practice in a condition that initially had only two foods. When the participant understood the task, a new food was added, until the practice condition replicated the 1-screen condition with five foods. The task was administered in the fixed order of 1 -screen, 2-screen, and 6-screen conditions, each one preceded by practice for that display type.

The program recorded all the responses in a detailed time line as the participant completed each condition. These sequences were used to calculate several measures whose interpretation was suggested speculatively by Craik and Bialystok (2006). The first variable represented the disparity between the time required to cook that food and the actual time the food was cooked. This variable, discrepancy, was calculated as the absolute value of the difference in time between the actual and required cooking times and then averaged across the five foods. Typically, discrepancy values reflected late stopping times, that is, the length of time that the foods were overcooked. This variable indicates prospective memory through the ability to remember to stop each food at the right time.

The second measure was the range of stop times and indicated the difference in seconds between stopping the first and the last foods. Ideally, this number should approach 0 because participants were told that an important goal was to have all the foods finish cooking at the same time. This variable reflects planning ability and, to some extent, working memory.
The third measure was the deviation of start times. To achieve a low range of stop times, participants had to calculate ideal start times for each food adjusted according to the previous start times. For example, if the second food should be started $90 \mathrm{~s}$ after the first food but was not started until $110 \mathrm{~s}$ had elapsed, there would be no perfect time to start the third food. In order for the foods to finish cooking at the same time, the third food should be started $120 \mathrm{~s}$ after the first food and $30 \mathrm{~s}$ after the second food. The best that could be done in this case, however, is the average between $120 \mathrm{~s}$ (for Food 1) and $110+30=140 \mathrm{~s}$ (for Food 2); that is, Food 3 should ideally be started $130 \mathrm{~s}$ after Food 1. The measure calculated for each participant was the average deviation between the ideal and actual start times for Foods 2 to 5. This variable provides a further indicator of planning ability, as well as a component of prospective memory.

Fourth, the number of times that participants checked the progress of foods when they switched screens was calculated for the 2- and 6-screen conditions. Monitoring participants' checking was not possible in the 1-screen condition. This variable indicates planning.

The fifth measure indicated the proportion of task time spent at table setting and had two components. The first was the average time per condition spent setting the table expressed as the proportion of total task time. The second was inappropriate table setting, defined as the amount of time spent setting the table when the cooking required attention, specifically, because an ideal start time or stop time had passed. This measure was expressed as the proportion of table setting time that should have been used to attend to cooking. In our view these variables indicate task switching and perseveration (Craik \& Bialystok, 2006).

\section{RESULTS}

The background measures for all the participants, shown in Table 1, were each analyzed by a 2-way ANOVA for group and gender. Apart from the obvious difference in age between young controls and the other two groups, there was no age

Table 1. Mean age, number of years of formal education, and score on each of the background measures (and standard deviation) for participants in the three groups by gender. Mean scores collapsed across gender are indicated in italics.

\begin{tabular}{|c|c|c|c|c|c|c|c|}
\hline Group & Gender & $\mathrm{N}$ & Age & Education & Mill Hill & Alpha span & Digit span \\
\hline \multirow[t]{3}{*}{ Younger } & Female & 10 & $21.4(2.7)$ & $13.9(2.2)$ & $19.4(3.5)$ & $32.6 \quad(7.5)$ & $60.10(20.6)$ \\
\hline & Male & 5 & $20 \quad(3.2)$ & $13.6(1.3)$ & $19.8(2.5)$ & $39.2 \quad(6.5)$ & $78.8 \quad(25.6)$ \\
\hline & Mean & 15 & $20.9(2.7)$ & $13.8(1.9)$ & $19.5(3.1)$ & 34.8 (7.6) & 66.3 \\
\hline \multirow[t]{3}{*}{ Older } & Female & 10 & $70.3(5.1)$ & $15.3(2.7)$ & $24.5(3.3)$ & $32.6(13.0)$ & $64.2 \quad(22.5)$ \\
\hline & Male & 5 & $70.5(2.8)$ & $17.8(4.8)$ & $23.2(3.7)$ & $35.2(13.2)$ & $(32.8)$ \\
\hline & Mean & 15 & $70.4(4.4)$ & $16.1(3.5)$ & $24.1(3.4)$ & $33.5(12.6)$ & $71.8 \quad(27.5)$ \\
\hline \multirow[t]{3}{*}{$\mathrm{PD}$} & Female & 8 & $67.8(6.9)$ & $15.5(3.5)$ & $23.4(4.8)$ & $31.9(10.5)$ & $66.5 \quad(23.6)$ \\
\hline & Male & 9 & $69.1(8.1)$ & $16 \quad(4.1)$ & $21.6(5.9)$ & $26.6(9.6)$ & (21.1) \\
\hline & Mean & 17 & $68.5(6.0)$ & $15.8(3.7)$ & $22.4(5.3)$ & $29.1(10.1)$ & 62.5 (21.9) \\
\hline
\end{tabular}




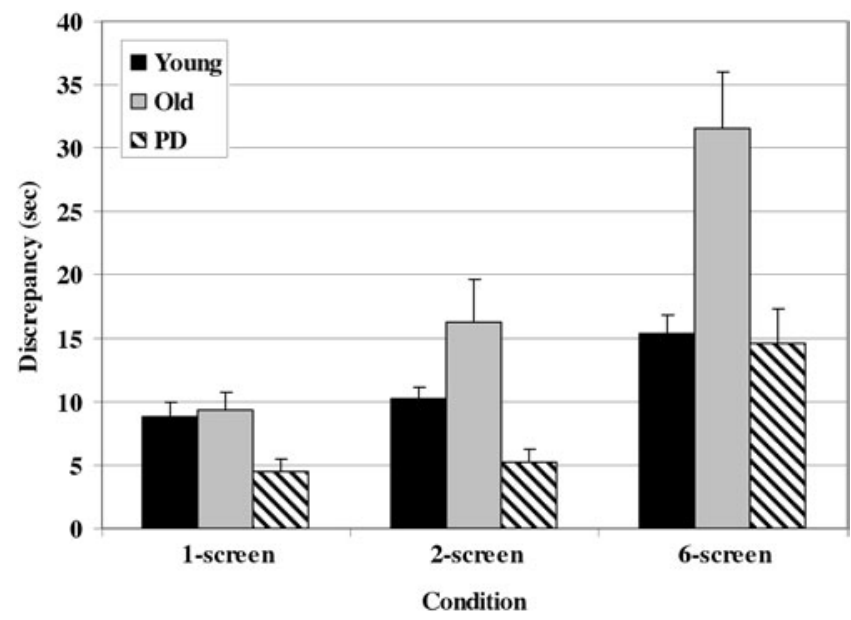

Fig. 2. Mean discrepancy scores (and standard errors) between specified and actual cooking times by group and condition.

difference between the older controls and the PD patients, $t(30)=1.4$, n.s. The only variable for which there was a significant group difference was Mill Hill Vocabulary score, $F(2,41)=4.44, p<.02$, in which the older control adults obtained higher scores than the young control group, with the PD patients midway between and not different from either. There was no effect of gender or interaction of group with gender in any of the analyses. The finding that PD patients did not differ from the older control group (or even from the younger group) supports the claim for their mental competence.

The discrepancy score indicating the mean number of seconds that the food was overcooked is shown in Figure 2. A 2-way ANOVA for condition (3) and group (3) revealed effects of condition, $F(2,88)=42.19, p<.0001$, group, $F(2,44)=10.97, p<.0001$, and their interaction, $F(4,88)=$ $5.28, p<.001$. For the 1 -screen condition, Scheffé tests showed that there was no difference between the young and old control groups but the PD group had significantly smaller discrepancy scores than either of them; for the 2-screen condition, the PD group had smaller discrepancy scores than the older adults but the young adults were not different from either of these; for the 6-screen condition, the PD and young adult group had the smallest discrepancy scores, and both of these were significantly smaller than those for the older adults. The increase in discrepancy across the conditions was also different for the three groups: The young and old control groups began with the same score in the simplest condition, but the older adults became progressively less accurate as the conditions became more difficult. The PD group began with the smallest discrepancy score and by the most difficult condition, had reached the level of the young adults.

The data for the range of stop times are shown in Figure 3. A 2-way ANOVA for condition and group indicated main effects for condition, $F(2,88)=6.59, p<.003$, and group, $F(2,44)=3.51, p<.04$, with no interaction. The 6 -screen condition produced a greater range than either the

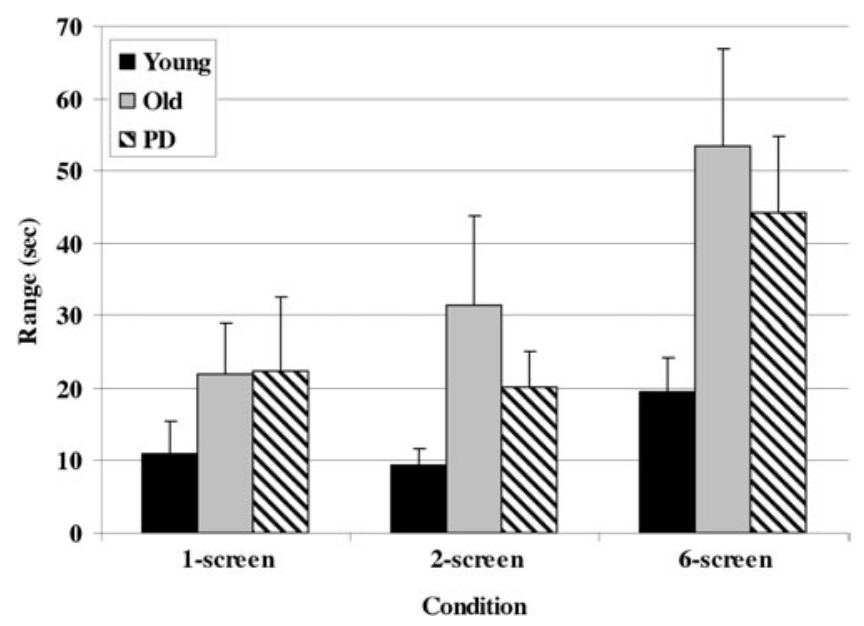

Fig. 3. Mean range of stopping times between first and last food stopped (and standard errors) by group and condition.

1 -screen or 2-screen conditions, with no difference between these two, and the older participants had a larger range of stop times than younger participants, with the PD patients not different from either of these. Although the interaction was not significant, the results appeared different for the three scenarios so they were also analyzed individually. There was no difference between groups for either the 1-screen, $F<1$, or 2 -screen, $F(2,44)=1.98$, n.s., conditions, but there was an effect for the 6-screen condition, $F(2,44)=$ $3.39, p<.05$, caused by a difference between the young and old participants $(p<.02)$ with the PD patients not different from either.

The calculation of the discrepancy from the optimal starting time given the progress of the other foods is shown in Figure 4. A 2-way ANOVA revealed an effect of condition, $F(2,86)=13.67, p<.0001$, in which the 6-screen condition elicited larger discrepancies than the other two, and an effect of group, $F(2,43)=3.67, p<.03$, in that the older

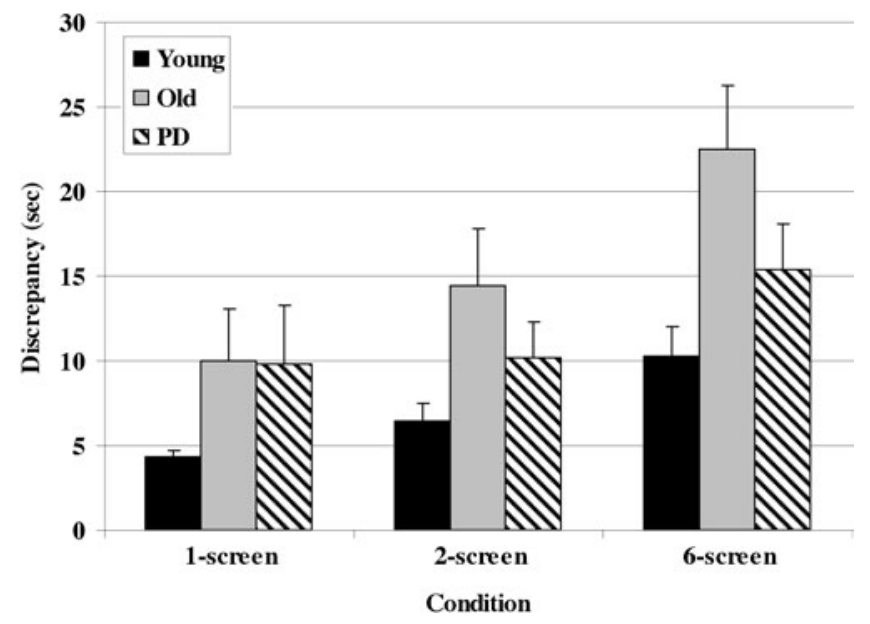

Fig. 4. Mean deviation of start times from ideal start times (and standard errors) by group and condition. 
Table 2. Mean number of places set (and standard deviations) for the three groups in the 1-screen, 2-screen, and 6-screen conditions

\begin{tabular}{llcccc}
\hline \hline & $\mathrm{N}$ & 1-Screen & 2-Screen & 6-Screen & Average \\
\hline Younger control & 15 & $47.3(8.8)$ & $47.6(9.8)$ & $48.8(8.5)$ & $48.0(8.4)$ \\
Older control & 15 & $32.7(7.8)$ & $31.5(8.5)$ & $31.1(9.8)$ & $31.8(8.3)$ \\
PD patients & 17 & $27.0(11.2)$ & $25.4(8.8)$ & $23.5(9.2)$ & $25.4(9.1)$ \\
\hline \hline
\end{tabular}

adults produced larger discrepancies than the young adults, with the PD patients between these groups and not significantly different from either. The group by condition interaction was not significant.

The mean numbers of checks for the 2- and 6-screen conditions are shown in Figure 5. Although more checks were made in the 6 -screen condition, $F(1,44)=63.06, p<$ .0001 , there was no difference in the number of checks carried out by the three groups, $F(2,44)=2.88, p=.07$, and no group by condition interaction.

The numbers of places set in each condition are shown in Table 2. A 2-way ANOVA found a strong effect of group, $F(2,44)=28.60, p<.01$, but no effect of condition and no interaction $(F \mathrm{~s}<1.6)$. The young group set more places $($ mean $=48.0)$ than the older group $($ mean $=31.8)$, who in turn set more places than the PD group (mean $=25.4)$, with all inter-group differences significant at $p<.01$. The proportion of task time spent setting the table and the proportion of that time that was inappropriate because one of the foods required attention are displayed in Figure 6. For the proportion of time spent overall, a 1-way ANOVA indicated an effect of group, $F(2,44)=23.94, p<.0001$, in which there was no difference between the young and old adults but the PD patients spent significantly less time on table setting than the other two groups (Scheffé, $p<.05$ ). Similarly, the amount of time spent on table setting that was inappropriate also yielded an effect of group, $F(2,44)=$

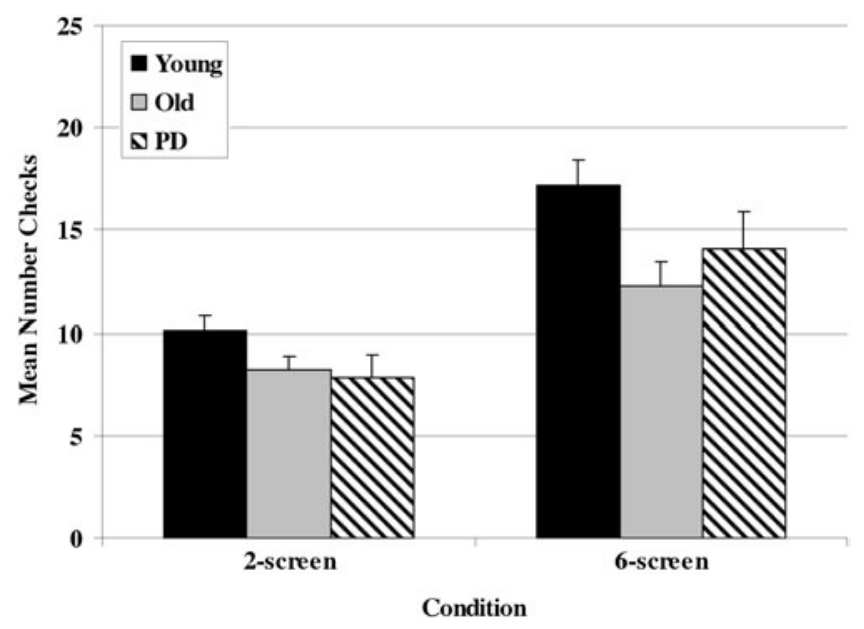

Fig. 5. Mean number of food checks (and standard errors) by group and condition.
$11.87, p<.0001$, but this time because the older adults spent more inappropriate time than either the young adults or PD patients who were not different from each other.

The average time taken to complete each place setting was also calculated for each group. These values are $4.8 \mathrm{~s}$, $8.4 \mathrm{~s}$ and $6.1 \mathrm{~s}$ for the younger, older, and PD groups respectively. A 1-way ANOVA yielded a significant effect of group, $F(2,44)=10.81, p<.001$, and Scheffé contrasts showed that both young and PD groups took less time per setting than the older group, but that the difference between the young and PD groups failed to reach significance $(p=.09)$. The important result is that the PD group carried out the table setting task more rapidly than the age-matched control, indicating that the patients were not slowed by possible motor difficulties.

An ANCOVA on the three measures of cooking accuracy was conducted using as covariates the two table-setting task variables that were significantly different across groups, namely, number of places set and proportion of time spent in inappropriate table setting. The purpose was to establish whether the group difference on the primary cooking variables remained once the different levels of performance in the secondary task were taken into account. The dependent variables were the averages across the three conditions of discrepancy, stop ranges, and start time scores, rather than the individual scores for each condition. The analysis showed a significant difference between groups for discrepancy scores, $F(2,42)=11.53, p<.0001$, and range of stop times, $F(2,42)=3.90, p<.03$, but not for adjusted start times, $F<1$. For both discrepancy scores and stopping range, the PD patients had smaller deviations than the other two groups, with no significant difference between the two control groups (see Table 3).

Table 3. Least square means in seconds for discrepancy scores, range of stopping times, and deviation of start times from ideal start times with standard error, covarying out proportion of time spent in inappropriate table setting and average number of places set. Values for all variables are the average across the three conditions.

\begin{tabular}{lccc}
\hline \hline Group & Discrepancy & $\begin{array}{c}\text { Range of } \\
\text { stop times }\end{array}$ & $\begin{array}{c}\text { Ideal } \\
\text { start times }\end{array}$ \\
\hline Younger control & $13.49(2.4)$ & $27.35(7.9)$ & $11.03(3.1)$ \\
Older control & $18.65(1.7)$ & $32.71(5.9)$ & $10.47(2.3)$ \\
PD patients & $6.68(1.9)$ & $19.08(6.6)$ & $12.85(2.6)$ \\
\hline \hline
\end{tabular}




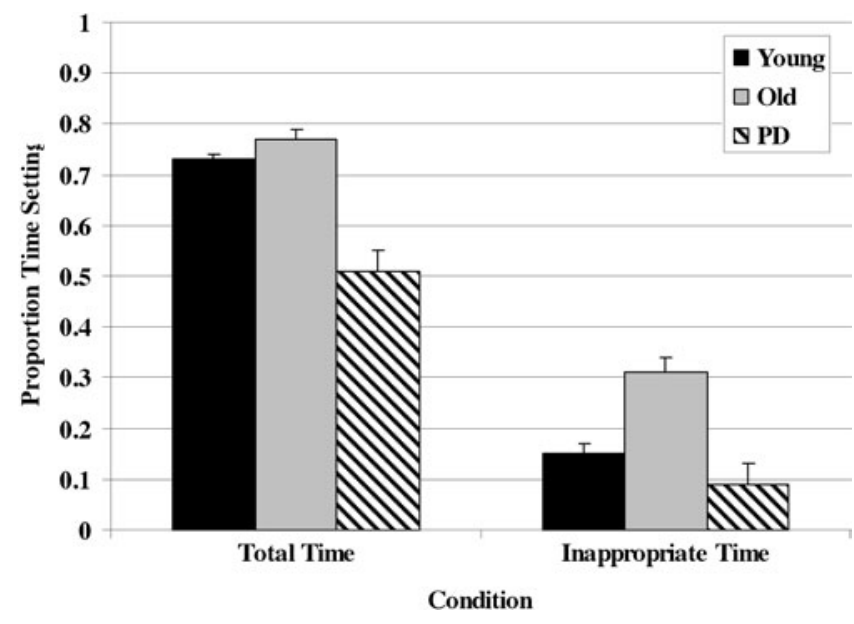

Fig. 6. Mean proportion of time (and standard error) spent on table-setting task as a proportion of total time spent on task and proportion of time inappropriately spent on table-setting because a food needed to be started or stopped.

Finally, correlations were calculated to determine the relation between performances on the task components. Taking discrepancy scores and range of stop times as measures of cooking efficiency and numbers of places set as a measure of table-setting performance, there was a marginally significant negative correlation between places set and discrepancy for the PD patients, $r(15)=-0.45, p=0.07$, but the corresponding correlations were low and non-significant for both the young group, $r(13)=+0.10$, n.s., and the old group, $r(13)=-0.14$, n.s. Similarly, the PD patients showed a significant negative correlation between places set and range of stop times, $r(15)=-0.61, p<0.01$, whereas this relation was again absent in both young, $r(13)=-0.02$, n.s., and old groups, $r(13)=-0.25$, n.s. Thus, the Parkinson's patients who performed particularly well on the cooking task did so at the cost of carrying out the tablesetting component of the overall task.

\section{DISCUSSION}

Given that performance on the breakfast task requires substantial amounts of planning and executive control functions, and that PD patients are impaired on these functions, we expected to find large deficits in their performance relative to age-matched controls. Surprisingly however, the patients outperformed controls on most measures, and often matched the performance levels of young adults. The Parkinson's patients tested in this study were high functioning and well matched with the monolingual older adults reported in the original study (Craik \& Bialystok, 2006) in terms of age, and with both the younger and older control groups in terms of education, short-term memory, and working memory. It is not surprising that their performance on the breakfast task was different from that of participants in the two healthy control conditions; what is surprising is the direction of that difference.
On the three primary measures of cooking accuracy, namely, deviation from exact cooking time, range of stop times, and deviation from optimal starting time, the younger controls were consistently better than the older controls. The PD patients, although showing slightly different results for each measure, were never significantly worse than the younger controls and always better than the older controls. For the discrepancy from actual cooking time, the most straightforward measure of accuracy, the patients were better than the young controls on the simplest condition and equivalent to the young controls on the most difficult one. On the other two accuracy measures, the patients were partway between the younger and older control groups and not significantly different from either. The three accuracy measures reflect important aspects of executive control—prospective memory, planning, switching, and working memory - all of which are known to be impaired in Parkinson's patients. How, then, could the PD patients do at least as well as and in some cases better than age-matched controls? One possibility is that the two groups approached the task with different strategies.

One indication of strategy is the monitoring of the cooking task provided by the number of food checks. Although this is a rough measure and indicates only when some update on progress was obtained, it provides an overall indication of the extent to which the participants were continually aware of the need to track the cooking times. The interesting result for this measure is that there were no group differences; all participants monitored cooking progress with the same number of updates.

The explanation for the high accuracy of the Parkinson's patients likely comes from the last set of variables, namely the number of places set, the proportion of task time spent at table setting, and the proportion of that time that was misspent because one of the foods required attention. The Parkinson's patients simply spent less time on the tablesetting task than the older adults, setting significantly fewer places and reallocating the time to cooking. Thus, these participants were more vigilant of the time each food had left to cook and were more accurate in pressing the stop button. At the same time, they were more conservative in their decision to perform the table-setting task, switching to it only when they were confident that the foods did not require attention. It should be noted that their decision to set fewer places is not attributable to possible motor problems; in fact the PD patients took significantly less time to set each place than participants in the older control group. Their different pattern of performance apparently reflects a cognitive preference to concentrate on the cooking task while spending less time on table setting. Moreover, the higher performance levels of the PD group relative to their agematched controls were maintained for two of the measures (discrepancy and range of stopping times) even after controlling for differences in table-setting performance by ANCOVA. The PD patients apparently exhibited superior performance on a task involving cognitive flexibility.

Recent findings and theoretical accounts in the PD literature suggest that the pattern of performance shown by the 
PD patients may be less attributable to a voluntary strategy than to a consequence of specific cognitive deficits. It seems that the neurobiological changes in PD (in particular, striatal dopamine depletion) do not simply disrupt functioning but rather shift the system in favor of one mode at the expense of another. For example, Gauntlett-Gilbert and colleagues (1999) found that PD patients were more impaired on extra-dimensional shifts than on intra-dimensional shifts in a set-shifting task, and concluded that PD was associated with "an inflexible exaggeration of selective attention" (Gauntlett-Gilbert et al., 1999, p. 615). Lewis and colleagues (2005) confirmed the finding that PD patients were disproportionately impaired on extra-dimensional shift tasks. Converging conclusions were drawn by Crofts and colleagues (2001) from work on marmosets. These researchers found that dopamine depletion in the caudate was associated with exaggerated focusing on currently relevant stimuli and a concomitant reduction of responding to alternative stimuli. Finally, Cools and colleagues (Cools, 2006; Cools et al., 2001) concluded that mild PD is associated with an impairment of a selection mechanism that is necessary for disengaging from a current task set and engaging in a new task set in the face of distraction. Based on these findings and conclusions, we suggest that the PD patients in the present study were similarly more "stimulus-bound" by the more interesting and seemingly important cooking aspects, and less likely to switch temporarily to the tablesetting task. That is, PD patients may deal with their welldocumented problems in executive control by focusing more exclusively on the perceived main task. This relative neglect of one task in dual-task situations has also been reported in older adults. Broadbent and Heron (1962) required participants to perform a visual letter cancellation task while monitoring a series of auditory letters for a repeated letter. Young adults performed both tasks reasonably well, but older adults tended to concentrate on one task while performance on the other task suffered. This interpretation is supported by the pattern of correlation between the task components, as reported earlier.

Given that the patients performed less well on the tablesetting aspects of the task, it is not strictly the case that they showed an advantage over their age-matched peers. We are claiming, rather, that they behaved adaptively by concentrating on the primary task, thereby diminishing the overall need for prospective memory, working memory, and task switching. It is unclear whether this adaptive behavior reflects a deliberate strategy, or occurred as a consequence of their PD-induced tendency to weight important situations more highly than their counterparts and thus exhibit a set-shifting "deficit." However caused, this behavior of task simplification involving focusing and selective ignoring has implications for cognitive rehabilitation approaches for patients with Parkinson's disease. An approach that attempts to simplify the context in which tasks are carried out and focuses attention on individual parts of a problem instead of simultaneously managing a range of demands may prove to be an effective way to improve performance in Parkinson's patients. Although many rehabilitation approaches incorporate this strategy, the evidence from the present study establishes its effectiveness in dealing with complex tasks. A limitation of the study is that the performance of the PD patients may reflect their medication as much as their condition. However, it was not possible to test the participants without their medication because the fine-motor control demands of the task may have been too great. Thus, the results need to be extended in future studies that compare patients on and off medication.

\section{ACKNOWLEDGMENTS}

This work was supported by a grant from the Canadian Institutes of Health Research. We thank Jane Logan and Jeni Pathman for their assistance in completing the study. We are grateful to Dr. Patrick Davidson for help in contacting patients and to an anonymous reviewer for suggestions regarding interpretation.

\section{REFERENCES}

Braver, T.S. \& Barch, D.M. (2002). A theory of cognitive control, aging cognition, and neuromodulation. Neuroscience \& Biobehavioral Reviews, 26, 809-817.

Broadbent, D.E. \& Heron, A. (1962). Effects of a subsidiary task on performance involving immediate memory in younger and older men. British Journal of Psychology, 53, 189-198.

Cools, R. (2006). Dopaminergic modulation of cognitive functionimplications for L-DOPA treatment in Parkinson's disease. Neuroscience and Biobehavioral Reviews, 30, 1-23.

Cools, R., Barker, R.A., Sahakian, B.J., \& Robbins, T.W. (2001). Mechanisms of cognitive set flexibility in Parkinson's disease. Brain, 124, 2503-2512.

Craik, F.I.M. (1986). A functional account of age differences in memory. In F. Klix \& H. Hagendorf (Eds.), Human memory and cognitive capabilities, mechanisms, and performances (pp. 409-422). Amsterdam: Elsevier.

Craik, F.I.M. \& Bialystok, E. (2006). Planning and task management in older adults: Cooking breakfast. Memory and Cognition, 34, 1236-1249.

Crofts, H.S., Dalley, J.W., Collins, P., Van Denderen, J.C.M., Everitt, B.J., Robbins, T.W., \& Roberts, A.C. (2001). Differential effects of 6-OHDA lesions of the frontal cortex and caudate nucleus on the ability to acquire an attentional set. Cerebral Cortex, 11, 1015-1026.

Culbertson, W.C., Moberg, P.J., Duda, J.E., Stern, M.B., \& Weintraub, D. (2004). Assessing the executive function deficits of patients with Parkinson's disease: Utility of the Tower of London-Drexel. Assessment, 11, 27-39.

Dagher, A., Owen, A.M., Boecker, H., \& Brooks, D.J. (2001). The role of the striatum and hippocampus in planning: A PET activation study in Parkinson's disease. Brain, 124, 1020-1032.

Daniels, K., Toth, J., \& Jacoby, L. (2006). The aging of executive functions. In E. Bialystok \& F.I.M. Craik (Eds.), Lifespan Cognition: Mechanisms of Change (pp. 96-111). New York: Oxford University Press.

Dirnberger, G., Frith, C.D., \& Jahanshahi, M. (2005). Executive dysfunction in Parkinson's disease is associated with altered pallidal-frontal processing. Neuroimage, 25, 588-599.

Dubois, B. \& Pillon, B. (1997). Cognitive deficits in Parkinson's disease. Journal of Neurology, 244, 2-8. 
Edwards, D. \& Ryan, J.D. (2004). Identifying the Real Life Implication of Mild Stroke. Paper given at the American Congress of Rehabilitation Medicine-American Society of Neurorehabilitation (ACRM-ASNR) Joint Annual Conference, September 2004 in Ponte Vedra, FL.

Gauntlett-Gilbert, J., Roberts, R.C., \& Brown, V.J. (1999). Mechanisms underlying attentional set-shifting in Parkinson's disease. Neuropsychologia, 37, 605-616.

Johnson, A.M., Pollard, C.C., Vernon, P.A., Tomes, J.L., \& Jog, M.S. (2005). Memory perception and strategy use in Parkinson's disease. Parkinsonism and Related Disorders, 11, 111-115.

Kliegel, M., Phillips, L.H., Lemke, U., \& Kopp, U.A. (2005). Planning and realisation of complex intentions in patients with Parkinson's disease. Journal of Neurology, Neurosurgery \& Psychiatry, 76, 1501-1505.

Lewis, S.J., Cools, R., Robbins, T.W., Dove, A., Barker, R.A., \& Owen, A.M. (2003). Using executive heterogeneity to explore the nature of working memory deficits in Parkinson's disease. Neuropsychologia, 41, 645-654.

Lewis, S.J., Slabosz, A., Robbins, T.W., Barker, R.A., \& Owen, A.M. (2005). Dopaminergic basis for deficits in working memory but not attentional set-shifting in Parkinson's disease. Neuropsychologia, 43, 823-832.

Li, S.-C., Lindenberger, U., \& Frensch, P.A. (2000). Unifying cognitive aging: From neuromodulation to representation to cognition. Neurocomputing, 32-33, 879-890.
McNamara, P., Durso, R., Brown, A., \& Lynch, A. (2003). Counterfactual cognitive deficit in persons with Parkinson's disease. Journal of Neurology, Neurosurgery \& Psychiatry, 74, 1065-1070.

Muslimovic, D., Post, B., Speelman, J.D., \& Schmand, B. (2005). Cognitive profile of patients with newly diagnosed Parkinson disease. Neurology, 65, 1239-1245.

Owen, A.M., Sahakian, B.J., Hodges, J.R., Summers, B.A., Polkey, C.E., \& Robbins, T.W. (1995). Dopamine-dependent frontostriatal planning deficits in early Parkinson's disease. Neuropsychology, 9, 126-140.

Raven, J.C. (1960). Guide to the standard progressive matrices. London: H.K. Lewis.

Robbins, T.W., James, M., Owen, A.M., Sahakian, B.J., Lawrence, L.D., McInnes, L., \& Rabbitt, P.M.A. (1998). A study of performance on tests from the CANTAB battery sensitive to frontal lobe dysfunction in a large sample of normal volunteers: Implications for theories of executive functioning and cognitive aging. Journal of the International Neuropsychological Society, 4, 474-490.

Rubin, D.C. (1999). Frontal-striatal circuits in cognitive aging: Evidence for caudate involvement. Aging, Neuropsychology, and Cognition, 6, 241-259.

Woodward, T.S., Bub, D.N., \& Hunter, M.A. (2002). Task switching deficits associated with Parkinson's disease reflect depleted attentional resources. Neuropsychologia, 40, 1948-1955. 